TIT/HEP-330/COSMO-72

May, 1996

\title{
Compact hyperbolic universe and singularities
}

\author{
Akihiro ISHIBASHI⿵冂卄 \\ Department of Physics, \\ Tokyo Institute of Technology, Oh-Okayama, Meguro, Tokyo 152, Japan \\ Tatsuhiko KOIKEI \\ Department of Physics, \\ Keio University, Hiyoshi, Kohoku, Yokohama 223, Japan \\ Masaru SIINO用 \\ Department of Physics, \\ Kyoto University, Kyoto 606-01, Japan \\ Sadayoshi KOJIMA目 \\ Department of Mathematical and Computing Science, \\ Tokyo Institute of Technology, Oh-Okayama, Meguro, Tokyo 152, Japan
}

\begin{abstract}
Recently many people have discussed the possibility that the universe is hyperbolic and was in an inflationary phase in the early stage. Under these assumptions, it is shown that the universe cannot have compact hyperbolic time-slices. Though the universal covering space of the universe has a past Cauchy horizon and can be extended analytically beyond it, the extended region has densely many points which correspond to singularities of the compact universe. The result is essentially attributed to the ergodicity of the geodesic flow on a compact negatively curved manifold. Validity of the result is also discussed in the case of inhomogeneous universe. Relationship with the strong cosmic censorship conjecture is also discussed.
\end{abstract}

\footnotetext{
${ }^{*}$ E-mail: akihiro@th.phys.titech.ac.jp

${ }^{\dagger}$ E-mail: koike@rk.phys.keio.ac.jp

${ }^{\ddagger}$ E-mail: msiino@tap.scphys.kyoto-u.ac.jp, JSPS fellow

${ }^{\S}$ E-mail: sadayosi@is.titech.ac.jp
} 


\section{INTRODUCTION}

Recent observations of the density parameter $\Omega_{0} \approx$ 0.1 [1] suggest that the spatial curvature of the universe is negative. In this paper we call such the universe with negative curvature as a hyperbolic universe rather than a conventional open universe because the open universe is meant to be non-compact while we shall consider a compact negative curvature space.

On the other hand, it is believed that inflations occurred in the early stage of the universe because the Inflationary scenario is, so far, the only viable theory which can solve such cosmological problems as the flatness, horizon and monopole problems [2] without appealing to fine tuning. It is therefore of great interest to investigate hyperbolic inflationary universe scenarios.

There have been some works which studied the creation of the hyperbolic inflationary universe in the onebubble inflationary model [3].

During the inflation epoch, the universe is well approximated by a de Sitter space-time. Then, we notice that hyperbolic universe has no curvature singularity as long as it is in the inflationary phase, even though its spatial volume approaches to zero. The initial singularity is just a coordinate singularity. Hyperbolic inflationary universe is expected to be realized by taking hyperbolic time-slices on a portion of the de Sitter space-time. One example is the one-bubble inflationary scenario. In this context, any space-time which contains a hyperbolic inflationary universe has a past (Cauchy) horizon and continues further to the past because a hyperbolic chart cannot cover the whole de Sitter space-time.

Observations normally give us local information only such as the local spatial curvature of our universe, because local information is, in general, independent of global properties such as the topology of the universe. Some observational effects are, however, expected, if the periodicities due to the non-trivial topology are shorter than the horizon scale. Such possibilities have been studied in theoretical and observational cosmologies [4].

Compactness of the universe seems to be an acceptable and appealing notion, especially in the context of the canonical treatments of the universe (or quantum gravity). Compactness provides a finite value of the action integral and gives the natural boundary conditions for the matter and gravitational fields in the universe.

The notion of compactness of a given universe depends on how to take its spatial sections. We concentrate our interests on the case that hyperbolic hypersurfaces are compact. In particular, an intriguing question arises in the case that compact hyperbolic universe is in an inflationary phase. We naturally ask whether or not such universe has the past horizon and continues further to the past as the non-compact case. If it does, we expect that topology change takes place and closed timelike curves appear, which is suggested by the work on the Lorentzian topology change of $(2+1)$-dimensional com- pact black hole geometry by M. Siino [5]. If it does not, we may find restriction to possible topologies of the universe.

In this paper, we will study the spatially compact hyperbolic inflationary universe. We will find that the latter is indeed the case. Namely, we will show that compact hyperbolic universe cannot be extended beyond the past horizon. This means that if the universe is hyperbolic and was in an inflationary phase in the early stage, the universe is not spatially compact.

In the next section, we construct models of compact hyperbolic inflationary universe. In section 3, we examine the extendibility of the universe. Section 4 is for conclusions and discussions.

\section{COMPACT HYPERBOLIC INFLATIONARY UNIVERSE}

\section{A. Construction of compact universe}

In this section, we treat homogeneous isotropic models of hyperbolic universe in an inflationary phase. Such a model is described by a hyperbolic chart on 4-dimensional de Sitter space-time. In the hyperbolic chart, homogeneous isotropic spacelike hypersurface is a manifold $\mathrm{H}^{3}$ of constant negative curvature with isometry group $S O(3,1)$. This universe has a past Cauchy horizon, and in the extended region a homogeneous hypersurface becomes 3-dimensional de Sitter hypersurface $d S^{3}$, which is an orbit of $S O(3,1)$.

We construct the compact universe model by identifying points of the space-time. A compact hyperbolic hypersurface is given as the quotient space of $H^{3}$ by the discrete subgroup $\Gamma$ of its isometry group $S O(3,1)$. One of the simplest 3-dimensional compact hyperbolic manifold is known as the Seifert-Weber manifold [12], whose construction is explicitly shown in appendix A.

In general, the action of a group $\Gamma$ on a manifold $M$ must be properly discontinuous so that the quotient space $M / \Gamma$ be a Hausdorff manifold. We call an action of $\Gamma$ on $M$ properly discontinuous if it satisfies the following conditions [6,7];

1. each point $q \in M$ has a neighborhood $U$ such that $\gamma(U) \cap U=\emptyset$ for each $\gamma \in \Gamma$ which is not the identity element, and

2. if $q, r \in M$ are such that there is no $\gamma \in \Gamma$ with $\gamma(q)=r$, then there are neighborhoods $U$ and $U^{\prime}$ of $q$ and $r$ respectively such that there is no $\bar{\gamma} \in \Gamma$ with $\bar{\gamma}(U) \cap U^{\prime} \neq \emptyset$.

Condition (1) implies that the quotient $M / \Gamma$ is a manifold, and condition (2) implies that it is Hausdorff.

It is worth noting that, for a Riemannian manifold $(M, g)$, every discontinuous group $\Gamma$ of $\operatorname{Isom}(M, g)$ is 
properly discontinuous [7]. Here the action of $\Gamma$ on a Riemannian manifold is called discontinuous if for every $p \in M$ and every sequence $\left\{\gamma_{i}\right\}$ of elements of $\Gamma$, where all $\gamma_{i}$ are mutually distinct, the sequence $\left\{\gamma_{i}(p)\right\}$ does not converge to a point in $M[7]$. We can obtain the compact hyperbolic inflationary universe because the homogeneous hypersurface $H^{3}$ is a Riemannian manifold.

\section{B. Misner universe}

As the simplest example of a compact universe and its extension, let us see the construction of Misner universe, which is given as a quotient manifold in $(1+1)$ dimensions.

The Misner universe $\left(M, g_{M}\right)$ is a space-time diffeomorphic to $S^{1} \times \mathbf{R}$. Its metric is given by

$$
g_{M}=-t^{-1} d t^{2}+t d \psi^{2}, \quad t \in \mathbf{R}, \quad 0 \leq \psi \leq 2 \pi .
$$

The universal covering manifold is the region $(I, \widetilde{\eta})$ in Figure 1, which is a part of 2-dimensional Minkowski space-time $(\widetilde{M}, \widetilde{\eta})$. The isometry group of $(\widetilde{M}, \widetilde{\eta})$ is the Lorentz group $S O(1,1)$, whose orbits are the hyperbolae $\left(X^{0}\right)^{2}-\left(X^{1}\right)^{2}=: \sigma=$ constant. The covering transformation group $\Gamma$ of the Misner universe is a discrete subgroup of the Lorentz group consisting of $\gamma^{m}$, where $m$ is an integer and $\gamma$ maps $\left(X^{0}, X^{1}\right) \in \widetilde{M}$ to

$$
\left(\begin{array}{l}
X^{0^{\prime}} \\
X^{1^{\prime}}
\end{array}\right)=\left(\begin{array}{cc}
\cosh \pi & \sinh \pi \\
\sinh \pi & \cosh \pi
\end{array}\right)\left(\begin{array}{l}
X^{0} \\
X^{1}
\end{array}\right) .
$$

This subgroup $\Gamma$ acts on the region $(I, \widetilde{\eta})$ properly discontinuously. However, if we maximally extend the Misner universe, for any point $r$ on the null line $X^{0}+X^{1}=0$, the sequence $\left\{\gamma^{m}(r)\right\}$ has an accumulation point $o:=(0,0)$ on $(\widetilde{M}, \widetilde{\eta})$. Hence the condition (1) of properly discontinuous is not satisfied and the quotient $\widetilde{M} / \Gamma$ fails to be a manifold. We therefore must remove the accumulation point $o$ from the whole Minkowski space-time $(\widetilde{M}, \widetilde{\eta})$ so that we obtain the maximally extended Misner universe as a manifold $(\widetilde{M} \backslash\{o\}) / \Gamma$, which is illustrated in Figure 1. Since the action of $\Gamma$ on $\widetilde{M} \backslash\{o\}$ does not satisfy the condition (2), $(\widetilde{M} \backslash\{o\}) / \Gamma$ is a non-Hausdorff manifold.

Because of removing the point $o$ from $(\widetilde{M}, \widetilde{\eta})$, all causal curves toward $o$ are incomplete. We define a singularity as an incomplete causal curve according to Hawking and Ellis [6]. Thus, the maximally extended Misner universe has a singularity. This indicates that the existence of the accumulation points of action of $\Gamma$ causes singularities of the quotient space-time. In this paper, we call an accumulation point a topological singularity. The null boundaries of the original region $I$ and the extended regions $I I$, III are compact Cauchy horizons, which are generated by closed null geodesics.
FIG. 1. The maximally extended Misner universe is illustrated. The coordinate origin $o$ is an accumulation point of a sequence $\left\{\gamma^{m}(r)\right\}$. The region $I$ is the original Misner universe region. Under the action of $\Gamma$, points $s$ on the $\sigma$-constant surface are equivalent; similar for points $r$, points $q$, and points $t$, respectively. In the extended regions $I I, I I I$, closed timelike curves appear.

\section{ANOMALOUS OCCURRENCE OF SINGULARITIES}

In case of the compact hyperbolic inflationary universe model, the discrete subgroup $\Gamma$ of $S O(3,1)$ does not act on hypersurface $d S^{3}$ in the extended region properly discontinuously. Moreover, in contrast with the Misner universe, we will observe that topological singularities densely appear on the hypersurfaces $d S^{3}$ and the extended region is no longer a space-time manifold. Our main result is the following proposition.

\section{Proposition. \\ Suppose there exists an analytic extension of the universal cover of $(n+1)$-dimensional compact hyperbolic universe, where $n \geq 2$. Then, for every neighborhood $O_{c}$ of an ar- bitrary point $c$ in the $d S^{n}$, there exist $\gamma \in \Gamma$ and point $s \in d S^{n}$ such that an accumula- tion point $c^{\prime}$ of the infinite sequence $\left\{\gamma^{m}(s)\right\}$ is contained in $\mathrm{O}_{c}$.}

Under the assumption of the analyticity, we naturally observe that there exist a neighborhood which contains a Cauchy horizon in the hyperbolic inflationary universe and that in a Milne universe which are analytically diffeomorphic to each other. Here the Milne universe is the Minkowski space-time $E^{n, 1}$ with the hyperbolic chart. Thus it is convenient to exam in the Milne universe instead of the hyperbolic inflationary universe.

Since the argument is the same for $(n+1)$-dimensional universe with $n \geq 2$, we first present our argument in the $n=2$ case and comment on the $n \neq 3$ cases later.

Let us investigate what the action of $\Gamma$ is like in the extended region of the $(2+1)$-dimensional compact hyperbolic inflationary universe. As mentioned above, this 
is done in the 3-dimensional Milne universe. Let $E^{2,1}$ be a 3-dimensional Minkowski space-time with the metric

$$
\eta=-\left(d X^{0}\right)^{2}+\left(d X^{1}\right)^{2}+\left(d X^{2}\right)^{2} .
$$

In the model the hyperbolic hypersurface is 2-hyperbolic space $H^{2}$, which is embedded in $E^{2,1}$ as

$$
-\left(X^{0}\right)^{2}+\left(X^{1}\right)^{2}+\left(X^{2}\right)^{2}=-l^{2},
$$

by embedding $f: H^{2} \longrightarrow E^{2,1}$, where $l$ is the curvature radius. The induced metric is given by $g_{H}:=f^{*} \eta$ and whose isometry group is $S O(2,1)$.

In the extended region of the Milne universe the orbit of $S O(2,1)$ is $d S^{2}$, which is a surface

$$
-\left(X^{0}\right)^{2}+\left(X^{1}\right)^{2}+\left(X^{2}\right)^{2}=l^{2},
$$

in $E^{2,1}$. Each element of the subgroup $\Gamma$ must contain one of the boosts because otherwise the element consisted of only space-rotations would have fixed points. We consider the action of the element $\gamma_{\circ} \in \Gamma$ on $d S^{2}$, where $\gamma_{\circ}$ is represented in the coordinate system $\left(X^{0}, X^{1}, X^{2}\right)$ by the following matrix;

$$
\gamma_{\circ}(\zeta):=\left(\begin{array}{ccc}
\cosh \zeta & 0 & \sinh \zeta \\
0 & 1 & 0 \\
\sinh \zeta & 0 & \cosh \zeta
\end{array}\right), \quad \zeta=\text { constant } .
$$

$\gamma_{0}$ has three eigenvectors,

$$
a_{\circ}:=\left(\begin{array}{c}
1 \\
0 \\
-1
\end{array}\right), \quad b_{\circ}:=\left(\begin{array}{l}
1 \\
0 \\
1
\end{array}\right), \quad c_{\circ}:=l\left(\begin{array}{l}
0 \\
1 \\
0
\end{array}\right) .
$$

The two eigenvectors $a_{\circ}$ and $b_{\circ}$ are lying on the light cone,

$$
-\left(X^{0}\right)^{2}+\left(X^{1}\right)^{2}+\left(X^{2}\right)^{2}=0,
$$

and the point $c_{\circ}$ is in the $d S^{2}$ described by Eq. (3.3). Hereafter we use the same symbol to denote eigenvectors and the endpoints of the arrows of the eigenvectors. As in the maximally extended Misner universe, we observe that any point $s$ on the null lines in the $d S^{2}$ through the point $c_{\circ}$ converges to $c_{\circ}$ by the action of $\gamma_{\circ}{ }^{m}$ as illustrated in Figure 2. That is, the point $c_{\circ}$ is the accumulation point of the infinite sequence $\left\{\gamma_{0}{ }^{m}(s)\right\}$. For any $\gamma\left(\neq \gamma_{0}\right) \in \Gamma$, $\gamma\left(c_{\circ}\right)$ is one of the eigenvectors of $\gamma \circ \gamma_{\circ} \circ \gamma^{-1} \in \Gamma$ and so the point $\gamma\left(c_{\circ}\right)$ in the $d S^{2}$ is also the accumulation point of the sequence $\left\{\left(\gamma \circ \gamma_{\circ} \circ \gamma^{-1}\right)^{m}(\gamma(s))\right\}$. Thus, there exist many, countably infinite, accumulation points of the sequences $\left\{\gamma^{m}(s)\right\}$ in the $d S^{2}$, if we consider all $\gamma \in \Gamma$.
FIG. 2. Orbits of $\gamma_{\circ}{ }^{m}(p), p \in d S^{2}$ are schematically depicted.

Now we demonstrate that the accumulation points, that are topological singularities, densely occur in $d S^{2}$.

First, for every point $c$ in the $d S^{2}$, there exist an element $\gamma_{c} \in S O(2,1)$ such that $c$ is one of the eigenvectors of $\gamma_{c}$. If $\gamma_{c} \in \Gamma$, there exists $s \in d S^{2}$ such that $c$ is the accumulation point of $\left\{\gamma_{c}^{m}(s)\right\}$.

In the case that $\gamma_{c} \notin \Gamma$, the other two eigenvectors, $a$ and $b$, of $\gamma_{c}$ are lying on the light cone (Eq.(3.6)). These two vectors uniquely determine a geodesic in the $H^{2}$, as depicted in Figure 3. In fact, these two eigenvectors span a plane $\Pi$ and the intersection $\Pi \cap H^{2}$ is an geodesic curve $\lambda$ in the $H^{2}$. The geodesic $\lambda$ is projected to a straight line $\lambda_{K}:=\Pi \cap D_{K}$ on the Klein Disk $D_{K}:=\left\{\left(X^{0}, X^{1}, X^{2}\right) \mid X^{0}=1,\left(X^{1}\right)^{2}+\left(X^{2}\right)^{2}<1\right\}$. The projection $\pi: E^{2,1} \ni\left(X^{0}, X^{1}, X^{2}\right) \mapsto\left(k_{1}, k_{2}\right) \in D_{K}$ is defined as

$$
k_{i}:=\frac{X^{i}}{X^{0}} \quad(i=1,2) .
$$

The induced metric takes the form

$$
\begin{aligned}
g_{K}=\frac{1}{\left(1-k^{2}\right)^{2}}\left[\left(1-{k_{2}}^{2}\right) d{k_{1}}^{2}\right. & +\left(1-{k_{1}}^{2}\right) d k_{2}{ }^{2} \\
& \left.+2 k_{1} k_{2} d k_{1} d k_{2}\right],
\end{aligned}
$$

where $k:=\sqrt{k_{1}{ }^{2}+k_{2}{ }^{2}}$. We can identify the $H^{2}$ and $D_{K}$ by the diffeomorphism $\pi \circ f$. This projection $\pi$ maps a geodesic curve of the $H^{2}$, which is a hyperbola in the $E^{2,1}$ to a straight line in the $E^{2,1}$. We can also identify the action of $\Gamma$ on the $H^{2}$ and the action of $\Gamma_{K}$ on $D_{K}$, where

$$
\gamma_{K}:=\pi \circ \gamma \circ \pi^{-1}, \quad \Gamma_{K}:=\left\{\gamma_{K} \mid \gamma \in \Gamma\right\} .
$$


FIG. 3. Projection $\pi: E^{2,1} \longrightarrow D_{K}$. The $H^{2}$ and $D_{K}$ is identified by $\pi \circ f$.

We can see that the straight line from $b$ to $a$ on $\bar{D}_{K}:=D_{K} \cup \partial D_{K}$ is the geodesic $\lambda_{K}$, where $\partial D_{K}:=$ $\left\{\left(X^{0}, X^{1}, X^{2}\right) \mid X^{0}=1,\left(X^{1}\right)^{2}+\left(X^{2}\right)^{2}=1\right\}$.

As long as considering the case that $\gamma_{c K} \notin \Gamma_{K}$, the geodesic $\lambda_{K}$ is not closed on $D_{K} / \Gamma_{K}$.

It can be observed that $\Xi:=\partial D_{K} \times \partial D_{K} \backslash \Delta_{\partial D_{K}}$ has one-to-one correspondence with the set of all geodesics on $D_{K}$, where $\Delta_{\partial D_{K}}:=\left\{(p, p) \mid p \in \partial D_{K}\right\}$. Namely, a pair $(a, b) \in \Xi$ can be identified with the geodesic on $D_{K}$ which has endpoints $a, b \in \partial D_{K}$.

The eigenvectors $a, b$ and $c$ are related to each other as follows. Choosing an appropriate coordinate system and a constant $\alpha$, the eigenvector $c$ can be represented as

$$
c=l\left(\begin{array}{c}
\sinh \alpha \\
\cosh \alpha \\
0
\end{array}\right)
$$

Then, the other two are uniquely determined as

$$
a=\left(\begin{array}{c}
\cosh \alpha \\
\sinh \alpha \\
-1
\end{array}\right), \quad b=\left(\begin{array}{c}
\cosh \alpha \\
\sinh \alpha \\
1
\end{array}\right)
$$

except for the freedoms of the norms. Accordingly, for any point $c \in d S^{2}$, a geodesic $\lambda$ on the $H^{2}$ (or a point in $\Xi)$ is uniquely determined. ${ }^{* *}$

Second, choose a Riemannian metric e $:=\left(d X^{0}\right)^{2}+$ $\left(d X^{1}\right)^{2}+\left(d X^{2}\right)^{2}$ on $E^{2,1}$. Take an open ball, $B_{c}(\delta):=$ $\left\{x \in E^{2,1} \mid\|x-c\|_{\mathbf{e}}<\delta\right\}$, of radius $\delta$ with respect to e and define a neighborhood of $c$ in the $d S^{2}$ as $O_{c}\left(\delta, d S^{2}\right):=$

\footnotetext{
** If the point $c$ is constrained on the part $\left\{\left(X^{0}, X^{1}, X^{2}\right) \mid\right.$ $\left.X^{0}>0\right\} \cup\left\{\left(X^{0}, X^{1}, X^{2}\right) \mid X^{0}=0,\left(X^{1}\right)^{2}+\left(X^{2}\right)^{2}=1\right.$, $\left.X^{1}>0\right\} \cup\{(0,0,1)\}$ of the $d S^{2}$, the point $c$ has one-to-one correspondence with a point in $\Xi$.
}

$B_{c}(\delta) \cap d S^{2}$. Similarly, define neighborhoods of $a$ and $b$ respectively in the $\partial D_{K}$ as $O_{a}\left(\delta, \partial D_{K}\right):=B_{a}(\delta) \cap \partial D_{K}$ and $O_{b}\left(\delta, \partial D_{K}\right):=B_{b}(\delta) \cap \partial D_{K}$ corresponding to $O_{c}\left(\delta, d S^{2}\right)$ (see Figure 4 ). Then, for any point $c^{\prime} \in O_{c}\left(\delta, d S^{2}\right)$, there exist $a^{\prime} \in O_{a}\left(\delta, \partial D_{K}\right), b^{\prime} \in O_{b}\left(\delta, \partial D_{K}\right)$ and $\gamma_{c^{\prime}} \in S O(2,1)$ such that $a^{\prime}, b^{\prime}$ and $c^{\prime}$ are the eigenvectors of $\gamma_{c^{\prime}}$.

FIG. 4. Neighborhoods of points $a, b \in \partial D_{K}$ and $c \in d S^{2}$.

Now, what we want to show is reduced to the following lemma;

\section{Lemma.}

For any $(a, b) \in \Xi$ and $\delta>0$, there exist $a^{\prime} \in O_{a}\left(\delta, \partial D_{K}\right), b^{\prime} \in O_{b}\left(\delta, \partial D_{K}\right), \gamma_{K} \in \Gamma_{K}$ and $p \in D_{K}$ such that

$$
\begin{gathered}
\lim _{n \rightarrow \infty} \gamma_{K}^{m}(p)=a^{\prime} \in O_{a}\left(\delta, \partial D_{K}\right) \\
\lim _{n \rightarrow-\infty} \gamma_{K}^{m}(p)=b^{\prime} \in O_{b}\left(\delta, \partial D_{K}\right) .
\end{gathered}
$$

Proof.

We show that on the compact hyperbolic manifold $D_{K} / \Gamma_{K}$ there exists a closed curve whose lift has endpoints $a^{\prime} \in O_{a}\left(\delta, \partial D_{K}\right), b^{\prime} \in O_{b}\left(\delta, \partial D_{K}\right)$ in $\partial D_{K}$. We can make such a closed curve by using a non-closed geodesic $\lambda_{K}(v):=\Pi \cap D_{K}$ affinely parameterized by $v$ and ergodicity of geodesic flow on a compact manifold with negative curvature (see appendix B). 
FIG. 5. A compact hyperbolic manifold $D_{K} / \Gamma_{K}$ with genus 2 is illustrated. $\lambda_{K}$ is a non-closed geodesic and $\mu$ is a line segment which connects two points $q$ and $r$ on the $\lambda_{K}$. Then, one can see a closed curve $(r \rightarrow p \rightarrow q \rightarrow r)$ composed of the line segment $(r \rightarrow p \rightarrow q)$ of the geodesic $\lambda_{K}$ and the line segment $\mu(q \rightarrow r)$.

Let $p$ be a point on $\lambda_{K}$ at $v=0$ and take an arbitrary small open neighborhood $O_{p}^{K}\left(\epsilon, D_{K} / \Gamma_{K}\right):=\{x \in$ $\left.D_{K} / \Gamma_{K} \mid d_{K}(x, p)<\epsilon\right\}$ of $p$, where $d_{K}$ is the distance naturally defined by $g_{K}$. From the ergodicity of geodesic $\lambda_{K}$, for any large $N>0$, there exist $v_{1}>N, v_{2}<-N$ such that $q:=\lambda_{K}\left(v_{1}\right) \in O_{p}^{K}$ and $r:=\lambda_{K}\left(v_{2}\right) \in O_{p}^{K}$ and the tangent vectors of $\lambda_{K}$ at these points $r, p, q$, are sufficiently parallel to each other as depicted in Figure 5. Connecting the points $r$ and $q$ by a suitable line segment $\mu$, we obtain a closed curve $(r \rightarrow p \rightarrow q \rightarrow r)$ on the $D_{K} / \Gamma_{K}$. Corresponding to this closed curve, there exists an element $\gamma_{K} \in \Gamma_{K}$.

FIG. 6. A lift $\widetilde{O}_{p}^{K}$ and its images $\gamma_{K}^{m}\left(\widetilde{O}_{p}^{K}\right)$ on $\widetilde{D}_{K}$.

Let us fix a component $\widetilde{O}_{p}^{K}$ of the lift of $O_{p}^{K}$ on $D_{K}$, which is diffeomorphic to $O_{p}^{K}$. Accordingly, $\tilde{p}, \tilde{q}, \tilde{r}$, $\lambda_{K} \cap \widetilde{O}_{p}^{K}$ and $\tilde{\mu}$ denote the corresponding lifts. Any component of the lift of $O_{p}^{K}$ is given by $\gamma_{K}^{m}\left(\widetilde{O}_{p}^{K}\right)$. These are illustrated in Figure 6. For any $N>0$ there exist $m_{1}, m_{2}>N, v_{1}>0$ and $v_{2}<0$ such that $\lambda_{K}\left(v_{1}\right) \in$ $\gamma_{K}^{m_{1}}\left(\widetilde{O}_{p}^{K}\right)$ and $\lambda_{K}\left(v_{2}\right) \in \gamma_{K}^{m_{2}}\left(\widetilde{O}_{p}^{K}\right)$.

Let $g_{E}$ denote the Euclidean metric on $\bar{D}_{K}$ induced by e. Then the radius of $\gamma_{K}^{m}\left(\widetilde{O}_{p}^{K}\right)$ measured by $g_{E}$ becomes smaller and smaller as $m \rightarrow \pm \infty$. We therefore have $\lim _{m \rightarrow \infty} \gamma_{K}^{m}\left(\widetilde{O}_{p}^{K}\right) \subset O_{a}\left(\delta, \partial D_{K}\right)$ and $\lim _{m \rightarrow-\infty} \gamma_{K}^{m}\left(\widetilde{O}_{p}^{K}\right) \subset O_{b}\left(\delta, \partial D_{K}\right)$. As a result, we obtain

$$
\begin{aligned}
a^{\prime} & :=\lim _{m \rightarrow \infty} \gamma_{K}^{m}(p) \in O_{a}\left(\delta, \partial D_{K}\right), \\
b^{\prime} & :=\lim _{m \rightarrow-\infty} \gamma_{K}^{m}(p) \in O_{b}\left(\delta, \partial D_{K}\right)
\end{aligned}
$$

Proof of the Proposition.

From the Lemma we immediately have the two eigenvectors $a^{\prime}$ and $b^{\prime}$ of $\gamma \in \Gamma$. Then we obtain the third eigenvector $c^{\prime}$ of $\gamma$ so that the point $c^{\prime}$, which is the accumulation point of $\left\{\gamma^{m}(s)\right\}$, is contained in $O_{c}\left(\delta, d S^{2}\right)$. This is the proof of $n=2$ case.

In $(3+1)$-dimensional case, the discrete subgroup of $S O(3,1) \cong \operatorname{Isom}\left(H^{3}\right)$ has four eigenvectors. Two of them are null vectors corresponding to $a$ and $b$ in the $(2+1)$ case. The other two corresponding to $c$ are spacelike and direct to points in the hypersurface $d S^{3}$. In addition, we can also observe the ergodicity of geodesic flows on three compact hyperbolic Riemannian manifold $H^{3} / \Gamma$ [1] (see appendix B). We immediately obtain the same result as the $(2+1)$-dimensional case; topological singularities densely occur in the $d S^{3}$.

The assertion is proven in a similar manner in the higher dimensional cases.

\section{CONCLUSIONS AND DISCUSSION}

We have demonstrated that if a space-time with spatially compact hyperbolic hypersurfaces is extended analytically by extending both the universal cover and the action of $\Gamma \subset \operatorname{Isom}(M)$ thereof, the topological singularities appear densely in the extended region and it is no longer a manifold. We conclude that the spatially compact hyperbolic inflationary universe cannot be extended beyond the Cauchy horizon of its universal cover. It follows that the universe cannot be compact, if the universe is hyperbolic and was in an inflationary phase. From observations, it is difficult to determine global properties of the universe. It is full of interest that our result theoretically answers to some global properties, for example, to the simple but fascinating question whether our universe is spreading infinitely or compact.

Our result that the universe cannot be extended analytically across the Cauchy horizon obviously holds in the case that the spatial sections are $\mathbf{R} \times H^{2} / \Gamma$, which is non-compact, or its quotient manifold $S^{1} \times H^{2} / \Gamma$. It is remarkable that this is true even if the metric is inhomogeneous along the $\mathbf{R}$ (or $S^{1}$ ) factor. Consequently, the 
universe with such a topology does not have a Cauchy horizon.

As the scenario for a birth of hyperbolic universe, onebubble inflationary universe scenario is an appealing one. However, it cannot be a universal covering manifold of a compact hyperbolic inflationary universe, because such a model has a past Cauchy horizon inside the bubble. Thus, one-bubble inflationary universe scenario is incompatible with a compact hyperbolic universe model. In other words, if the one-bubble inflationary scenario is verified by observations, our hyperbolic FriedmannRobertson-Walker (FRW) universe is not compact. In general, any scenario which realize the hyperbolic inflationary universe by inducing hyperbolic chart on a portion of de Sitter space-time has a Cauchy horizon for the hyperbolic hypersurfaces and hence it cannot be a universal cover of a compact hyperbolic inflationary universe.

As we show explicitly, our result holds for the $(2+1)$ dimensional case and anti-de Sitter space-time, compact 3-dimensional black hole geometry does not realize Lorentzian topology change [5].

One may say that this anomaly is due to the high degrees of space-time symmetry. However we can also discuss the case that there is no symmetry. The result is essentially attributed to the ergodicity of geodesics on compact manifold with negative curvature [11]. When we consider the inhomogeneous universe as a hyperbolic inflationary FRW universe model with perturbations on it, we can take a hypersurface whose sectional curvature is everywhere negative by taking a time-slice of sufficiently small scale factor, or $l$ in the Eq. (3.2) and Eq. (3.3), near the past Cauchy horizon of the background universe. If inhomogeneity due to fluctuations of matter fields is large enough, energy density of the matter fields dominates the universe and it is out of the context of the inflationary universe. In such a case, an initial curvature singularity appears instead of the past Cauchy horizon. Thus, even in the case that the considering universe model is inhomogeneous, we expect that if the Cauchy horizon exists in its universal cover there exists a neighborhood of the Cauchy horizon such that the hypersurfaces contained in it are everywhere negatively curved. Then we observe that this neighborhood is homeomorphic to the neighborhood of the Cauchy horizon in the Milne universe, as considered in the previous section. We therefore expect the same result of the Proposition even in the inhomogeneous case.

Our result that compact hyperbolic universe does not admit a Cauchy horizon is closely related to the strong cosmic censorship conjecture, which states that physically realistic space-time is globally hyperbolic [8,9]. The case of spatially compact, locally homogeneous vacuum models have been extensively investigated by P. T. Chruściel and A. D. Rendall [10]. Our result is not restricted to the vacuum case and our approach will be useful to resolve the issue in the case of inhomogeneous universe.

\section{ACKNOWLEDGMENTS}

We are grateful to Prof. A. Hosoya, Prof. H. Ishihara and Prof. T. Mishima for helpful discussions. T. K. and M. S. acknowledge financial supports from the Japan Society for the Promotion of Science and the Ministry of Education, Science and Culture.

\section{APPENDIX A: CONSTRUCTION OF THREE-COMPACT HYPERBOLIC MANIFOLD}

3-dimensional hyperbolic space $H^{3}$ can be embedded in 4-dimensional Minkowski space-time $E^{3,1}$ as

$$
-\left(X^{0}\right)^{2}+\left(X^{1}\right)^{2}+\left(X^{2}\right)^{2}+\left(X^{3}\right)^{2}=-1,
$$

where the curvature radius is normalized to unity. Taking the chart;

$$
\left\{\begin{array}{l}
X^{0}=\cosh \xi \\
X^{1}=\sinh \xi \cos \theta \\
X^{2}=\sinh \xi \sin \theta \cos \psi \\
X^{3}=\sinh \xi \sin \theta \sin \psi
\end{array}\right.
$$

the induced metric takes the form

$$
g_{H}=d \xi^{2}+\sinh ^{2} \xi\left(d \theta^{2}+\sin ^{2} \theta d \psi^{2}\right) .
$$

$\operatorname{Isom}\left(H^{3}\right)$ is $S O(3,1)$.

The simply connected Riemannian manifold $H^{3}$ can be compactified by quotienting by the subgroup $\Gamma$ of its isometry $S O(3,1)$. It is known that $H^{3}$ is tessellated by hyperbolic dodecahedra. This means that the fundamental region of $H^{3} / \Gamma$ is a hyperbolic dodecahedron [12]. Then the concrete representations of the generators of $\Gamma$ are given as the following six matrices $\left\{T_{i=1 \sim 6}\right\}$ under the coordinates $\left(X^{0}, X^{1}, X^{2}, X^{3}\right)$ of $E^{3,1}$;

$$
\begin{aligned}
& T_{1}:=B_{0-3}(\alpha) \circ R_{3}\left(\frac{3}{5} \pi\right), \\
& T_{2}:=R_{2}(2 \psi) \circ T_{1} \circ R_{2}(-2 \psi), \\
& T_{3}:=R_{3}\left(\frac{2}{5} \pi\right) \circ T_{2} \circ R_{3}\left(-\frac{2}{5} \pi\right), \\
& T_{4}:=R_{3}\left(\frac{4}{5} \pi\right) \circ T_{2} \circ R_{3}\left(-\frac{4}{5} \pi\right), \\
& T_{5}:=R_{3}\left(-\frac{4}{5} \pi\right) \circ T_{2} \circ R_{3}\left(\frac{4}{5} \pi\right), \\
& T_{6}:=R_{3}\left(-\frac{2}{5} \pi\right) \circ T_{2} \circ R_{3}\left(\frac{2}{5} \pi\right),
\end{aligned}
$$

where

$$
B_{0-3}(\alpha):=\left(\begin{array}{cccc}
\cosh \alpha & 0 & 0 & \sinh \alpha \\
0 & 1 & 0 & 0 \\
0 & 0 & 1 & 0 \\
\sinh \alpha & 0 & 0 & \cosh \alpha
\end{array}\right)
$$




$$
\begin{aligned}
R_{2}(2 \psi) & :=\left(\begin{array}{cccc}
1 & 0 & 0 & 0 \\
0 & \cos 2 \psi & 0 & \sin 2 \psi \\
0 & 0 & 1 & 0 \\
0 & -\sin 2 \psi & 0 & \cos 2 \psi
\end{array}\right) \\
R_{3}(\gamma) & :=\left(\begin{array}{cccc}
1 & 0 & 0 & 0 \\
0 & \cos \gamma & -\sin \gamma & 0 \\
0 & \sin \gamma & \cos \gamma & 0 \\
0 & 0 & 0 & 1
\end{array}\right) \\
\tanh \frac{\alpha}{2} & :=\frac{\sqrt{40 \sqrt{5}-75}}{5}, \quad \tan 2 \psi:=2
\end{aligned}
$$

The compact hypersurfaces $H^{3} / \Gamma$ are constructed by the identifications;

$$
X^{a} \stackrel{\text { identify }}{\longleftrightarrow} \quad X^{\prime b}=\left(T_{i}\right)^{a}{ }_{b} X^{b} .
$$$$
\text { * }
$$

FIG. 7. Gray parts of surfaces of dodecahedra are dihedral pieces with dihedral angle $2 \pi / 5$. The front surfaces of a hyperbolic dodecahedron are marked with the bold-faced letters $\mathbf{A} \sim \mathbf{L}$. The surfaces marked with the letters $\mathrm{A} \sim \mathrm{L}$ are behind. The dark gray triangular parts of the front surfaces are identified to the light gray triangular parts of the opposite side. Five dihedral pieces consistently meet at the identified edge.

The value of the boost angle $\alpha$ is determined such that the $H^{3} / \Gamma$ is a regular compact manifold. Each of the $T_{i}$ transforms each surface of a dodecahedron to an opposite side after rotating by $3 \pi / 5$. The rotations are necessary so that five dodecahedra with dihedral angle $2 \pi / 5$ consistently meet at the identified edge and add up to $2 \pi$ as depicted in Figure 7.

\section{APPENDIX B: ERGODIC THEORY}

A triplet $\left(M, \mu, \phi_{t}\right)$ is called an abstract dynamical system, where $(M, \mu)$ is a measure space and $\phi_{t}: M \rightarrow M$ is a one-parameter group of transformations which preserve measure $\mu$.

Let $f$ be a function on $M$. The time-average $f^{*}(x)$ of $f$ at $x \in M$ is defined as

$$
f^{*}(x):=\lim _{T \rightarrow+\infty} \frac{1}{T} \int_{0}^{T} f\left(\phi_{t}(x)\right) d t,
$$

which exists for almost every $x$ [11. The space-average $\bar{f}$ is defined as

$$
\bar{f}:=\frac{1}{\mu(M)} \int_{M} f(x) d \mu .
$$

A dynamical system $\left(M, \mu, \phi_{t}\right)$ is ergodic if almost everywhere $f^{*}(x)=\bar{f}$, for any $f$ which is integrable with respect to $\mu$ (i.e. $f \in L_{1}(M, \mu)$ ).

Let us derive a geometrical property implied by ergodicity. Let $\left(M, \mu, \phi_{t}\right)$ be an ergodic abstract dynamical system on a compact, connected Riemannian manifold $M$. Let $A$ be an open subset of $M$. Define a function $f_{A}$ as

$$
f_{A}(x):= \begin{cases}1, & x \in A \\ 0, & x \notin A .\end{cases}
$$

Then, there exists a time-average

$$
f_{A}^{*}(x)=\lim _{T \rightarrow \infty} \frac{I_{A}(T)}{T}
$$

for almost every $x$, where $I_{A}(T)$ is a total length of $t$ of the intersections of $\left\{\phi_{t}(x) \mid 0 \leq t \leq T\right\}$ and $A$. The space-average is given by

$$
\overline{f_{A}}=\frac{\mu(A)}{\mu(M)} .
$$

The ergodicity implies $f_{A}^{*}(x)=\bar{f}_{A}$ for any $A$ and almost every $x$. From these observations, we have the following lemma.

\section{Lemma.}

Let $\left(M, \mu, \phi_{t}\right)$ be an ergodic dynamical system. For any $T_{0}>0$, and for almost every $x$ and any open neighborhood $A$ of $x$, there exist $T>T_{0}$ such that $\phi_{T}(x) \in A$. 


\section{Proof.}

Suppose there does not exist $T>T_{0}$ such that $\phi_{T}(x) \in$ $A$, for almost every $x$. It follows from $(\mathrm{B} 1)$ that $f_{A}^{*}(x)=0$ for almost every $x$. On the other hand, (B2) implies that $\overline{f_{A}}>0$. Thus we have $f_{A}^{*} \neq \overline{f_{A}}$ for almost every $x$, which contradicts the ergodicity.

Let us consider a geodesic flow on a Riemannian manifold. Let $(M, g)$ be a compact, connected Riemannian manifold, and $T_{1} M$ be its unit tangent bundle. There is a natural Riemannian metric $\widehat{g}$ on $T_{1} M$ induced by $g$ and a natural measure $\mu$ on $T_{1} M$ induced by $\widehat{g}$. Consider a geodesic $\lambda_{x}$ on $M$ parametrized by length $\tau$ and, which determined by $x=(p, v) \in T_{1} M$ by the condition $\lambda_{x}(0)=p \in M, \dot{\lambda}_{x}(0)=v \in T_{1 p} M$. Each $\lambda_{x}$ has a unique lift on $T_{1} M$. Considering all geodesics we can define a geodesic flow $\phi_{\tau}$ on $T_{1} M$ by $\phi_{\tau}(x):=\left(\lambda_{x}(\tau), \dot{\lambda}_{x}(\tau)\right)$.

We essentially used the following theorem in the proof of the main proposition of this paper.

\section{Theorem.}

(Lobatchewsky-Hadamard-Anosov [1])

Let $M$ be a compact, connected Riemannian manifold with negative curvature. $T_{1} M$ be a unit tangent bundle of $M$. Then, the geodesic flow on $T_{1} M$ is ergodic.

From the Theorem and the Lemma above, we have the following, which is actually used in our proof.

\section{Proposition.}

Let $M$ be a compact, connected Riemannian manifold with negative curvature. Let $\lambda_{x}$ be a geodesic defined above. For any $T_{0}>0$, and for almost every $x=(p, v)$ and any open neighborhood $O$ of $p$ in $M$, there exist $T>T_{0}$ such that $\lambda_{x}(T) \in O$ and the (unit) tangent vector $\dot{\lambda}_{x}(T)$ is arbitrarily close to $\dot{\lambda}_{x}(0)$.

[1] M. Fukugita, et al., Astrophys. J.361, L1 (1990).

[2] A. H. Guth, Phys. Rev. D23, 347 (1981); K. Sato, M. N. R. A. S. 195, 467 (1981).

[3] S. Coleman and F. De Luccia, Phys. Rev. D21, 3305 (1980); J. R. Gott III, Nature 295, 304 (1982); J. R. Gott III and T. S. Statler, Phys. Lett. 136 B, 157 (1984); M. Sasaki, T. Tanaka, K. Yamamoto and J. Yokoyama, Phys. Lett. B317, 510, (1993); T. Tanaka, K. Yamamoto, M. Sasaki: How to make an open FRW universe $\sim$ Onebubble inflationary model, Proc. 4th Workshop on G. R. G (1994); T. Tanaka and M. Sasaki, Phys. Rev. D50, 6444 (1994); M. Bucher and A. S. Goldhaber, Phys. Rev. D52, 3314 (1995).

[4] L. Z. Fang and H. Sato, Gen. Rel. Grav. 17, 1117,(1985); M. Lachièze-Rey and J. P. Luminet, Phys. Rep. 254, 135214, (1995); R. Lehoueq, M. Lachièze-Rey and J. P. Lu- minet: Cosmic crystallography, preprint gr-qc/9604050 (April, 1996)..

[5] M. Siino, Class. Quantum Grav. 11, 1995 (1994).

[6] S. W. Hawking and G. F. R. Ellis: The Large Scale Structure Of Space-Time, Cambridge University Press, Cambridge (1973).

[7] S. Kobayashi and N. Nomizu: Foundations of Differential Geometry, I, Interscience Pub. (1963).

[8] R. Penrose, Riv. Nuovo Cim. 1, 252-276 (1969).

[9] V. Moncrief and D. Eardley, Gen. Rel. Grav. 13, 887-892, (1981).

[10] P. T. Chruściel and A. D. Rendall, Ann. phys. 242, 349, (1995).

[11] V. I. Arnold and A. Avez: Ergodic problems of classical mechanics, (Yoshioka-syoten (1972), in Japanese)

[12] Weber, C. and Seifert, H., Math. Z. 37, 237-253, (1933). 